\title{
OBITUARIO
}

\section{In Memoriam Doctor Marcel Gutiérrez-Correa (1952-2017)}

\section{In Memoriam Doctor Marcel Gutiérrez-Correa (1952-2017)}

\section{Gretty K. Villena}

Laboratorio de Micología y Biotecnología, Universidad Nacional Agraria La Molina. Avenida La Molina, Lima 15024, Perú Email Gretty K. Villena: gkvch@lamolina.edu.pe

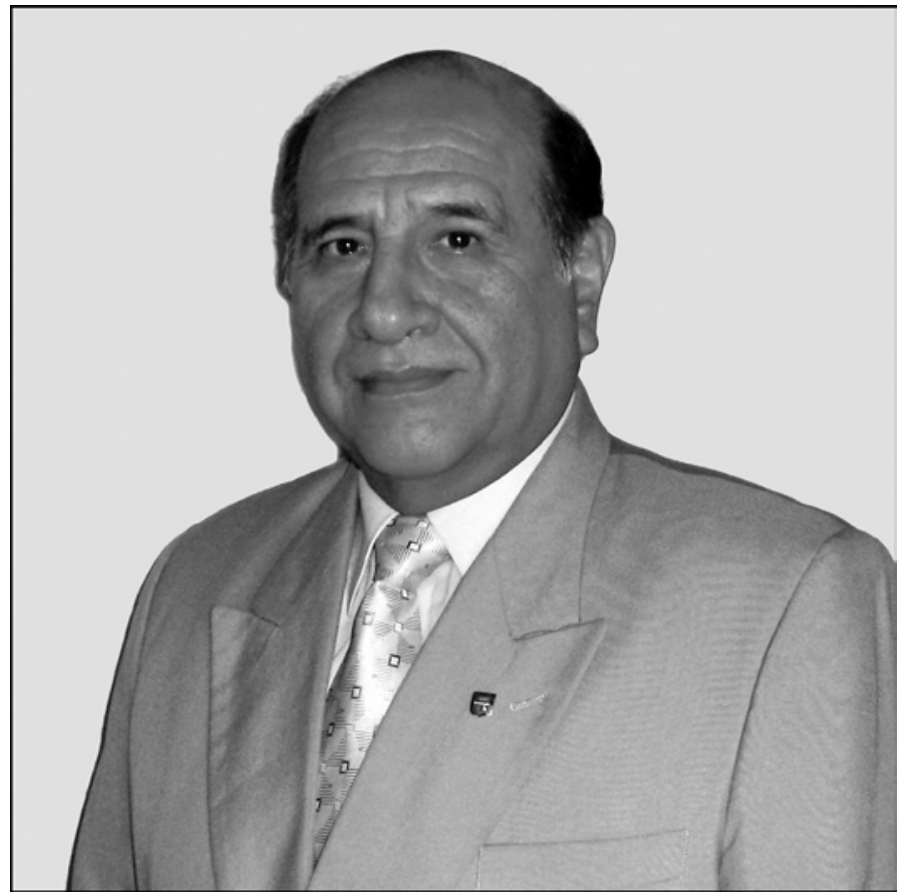

Doctor Marcel Gutiérrez-Correa

"Lo nuevo y lo desconocido son el motivo de la investigación y de la ciencia; el científico no teme a lo nuevo y a lo desconocido, lo enfrenta, lo clarifica, lo explica, abre camino"

El Doctor Marcel Gutiérrez-Correa, gran Maestro, reconocido científico, y Padre de la Biotecnología en el País, fue Profesor Principal del Departamento de Biología en la Facultad de Ciencias de la UNALM. Se graduó de Biólogo en la Universidad Nacional Agraria La Molina (UNALM) y obtuvo el grado de Doctor of Philosophy (Ph.D.) en Ciencias Agrícolas-Biotecnología por la Gifu University, Japón. Fue Fundador y Director hasta su partida, del Laboratorio de Micología y Biotecnología de la UNALM, dedicando su vida a la investigación científica durante 41 años ininterrumpidos. Fue Académico de Número en la Academia Nacional de Ciencias. Su excelencia académica y científica es equiparable a su calidad humana y grandeza de espíritu. Valoró su condición de Profesor Universitario, como un Título Nobiliario, al que honró hasta el final de sus días.

Este artículo está dedicado a la memoria del Profesor Marcel, con eterno agradecimiento, a quién fue mi Maestro y mi referente académico, y quién seguirá siendo a través de mi testimonio y el de sus alumnos, modelo de inspiración para las generaciones futuras.

\section{Citación:}

Villena G.V. 2017. In Memoriam Doctor Marcel Gutiérrez-Correa (19522017). Revista peruana de biología 24(2): 119 - 126 (Julio 2017). doi: http://dx.doi.org/10.15381/rpb.v24i2.13489 
El Profesor Gabriel Marcel Gutiérrez-Correa, nació en Piura el 8 de Enero de 1952, en el seno de una familia numerosa de la que fue el menor de ocho hermanos, entre quienes destacaron tanto el talento científico como artístico que no le fueron ajenos. Vivió orgulloso de su infancia en Piura y de su gusto por la gastronomía piurana, inculcado por sus padres en su negocio familiar.

Su grandeza espiritual y su compromiso de vida con la ciencia fueron sin duda, reflejo de su formación familiar, y constituyeron su sello personal, que dejó huella en muchas generaciones de jóvenes, para quienes fue modelo de inspiración.

\section{Su bistoria en la UNALM}

Su vocación científica despertó tempranamente, incluso desde sus juegos infantiles, y fue fortalecida por el ejemplo y admiración que sentía por su hermano mayor, el destacado médico e investigador en Bioquímica, Dr. José Gutiérrez Correa, ex Rector de la Universidad Nacional de San Agustín de Arequipa, a quién consideró su Maestro.

En el año, 1968, eligió a la Universidad Nacional Agraria La Molina (UNALM), como su Alma mater, ingresando a la carrera de Zootecnia, donde permaneció algunos meses para luego optar definitivamente por la carrera de Biología, incluso después de haber ingresado a la Facultad de Medicina de San Fernando de la Universidad Nacional Mayor de San Marcos. Sus estudios de pregrado transcurrieron en medio de un período de convulsión política nacional, de cisma en el gobierno de la UNALM, y de desastres telúricos que incluso destruyeron la Ciudad Universitaria. Pese a ello, logró ser un estudiante destacado, lo que le valió ser designado como Ayudante de Cátedra en el curso de Microbiología, aún siendo estudiante. Según su propio testimonio (Gutiérrez-Correa, 2012), sus referentes académicos fueron el Dr. Jacques Shonberg, profesor de Enzimología y a quién se refería como "sabio"; el Dr. Pedro Aguilar, quién fue su consejero académico y amigo entrañable; la Dra. Emma Loza, profesora de Citología y Fisiología Celular; el profesor Federico Anavitarte, quién lo inspiró con la frase "El profesor que no investiga da de beber agua estancada a sus alumnos"; y la Dra. Teresa Ames, prominente micóloga que influyó decisivamente en la elección de su línea de investigación.

En el año 1975 inició su carrera docente en la UNALM, como Jefe de prácticas del departamento de Biología, y en el año 1977, siendo aún Jefe de prácticas, formó el Laboratorio de Micología, posteriormente denominado Laboratorio de Micología y Biotecnología (1987), cuya trascendencia científica será detallada más adelante. Fue profesor auxiliar entre 1978 y 1981, profesor asociado hasta el año 1999 y finalmente Profesor principal desde el año 2000.

Como Profesor, fue una figura emblemática en la UNALM, respetado y admirado por muchas generaciones de alumnos. $\mathrm{Su}$ curso de "Fisiología Microbiana" del segundo currículo de la carrera de Biología (1973-1993) era uno de los mayores retos a superar durante la etapa de estudiantes debido a su rigurosidad científica, al uso de bibliografía muy actualizada y en idioma inglés y al incansable esfuerzo por rescatar el talento intelectual de sus alumnos en cada una de las preguntas que realizaba en clase y en el examen final del curso, que era oral y de más de una hora de duración. Era un compromiso estar a la altura de sus expectativas y demostrarle lo que se había aprendido e investigado adicionalmente sobre cada uno de los capítulos del curso. Luego, los cursos de Genética de Microorganismos y Microbiología Industrial, para quienes optaban la orientación en Microbiología, fueron la inspiración para despertar muchas vocaciones científicas.

En el tercer currículo de Biología (1993 a la fecha) fue profesor en la Orientación de Biotecnología, de los cursos de Genética Molecular Microbiana, Microbiología Industrial y Biotecnología, mostrando la misma rigurosidad científica y dedicación por formar a las nuevas generaciones de jóvenes, acorde a los estándares mundiales. Sus frases: "hay que ver más allá de lo evidente" y "la mejor respuesta es la más simple" han quedado grabadas en la mente de muchas generaciones.

Siempre fue visionario y adelantado a su época. Su labor en la UNALM, no se limitó a la docencia e investigación, también se involucró en la gestión académica. En el año 1988, consiguió financiamiento de la Corporación Andina de Fomento (CAF) a través del Programa Andino de Biotecnología, para iniciar el Programa Andino de Segunda Especialización en Biotecnología en la Facultad de Ciencias de la UNALM. Lamentablemente la propuesta no fue aceptada en el Consejo de Facultad y en 1989 la CAF entregó la subvención económica a un Instituto de Investigación en Venezuela.

Esto no lo amilanó y en el año 1992, siendo Presidente de la Comisión de Currículo de la Facultad de Ciencias, propuso al Departamento de Biología, la creación de la carrera de Ingeniería Biológica, adicional a la carrera de Biología. Su propuesta fue nuevamente rechazada. Sin embargo, la estructura curricular de dicha carrera sirvió de base para la reestructuración y aprobación en el año 1993 del Nuevo Currículo de Biología de la UNALM, vigente hasta la fecha, con dos orientaciones: Ecología y Biotecnología. Este nuevo enfoque de formación en biología, fue recogido y adoptado por otras Universidades en el país. En el año 1997, los alumnos de la primera promoción de este nuevo currículo solicitaron la implementación de la carrera de Ingeniería Biológica y el 2 de diciembre de ese año, el Consejo de Facultad de Ciencias aprobó la creación de la carrera y fue enviada para su ratificación en Consejo Universitario. Esto nunca ocurrió. Paradójicamente, hoy, 20 años después en el país se ofrecen exitosamente carreras como Bioingeniería, Ingeniería Biomédica y otras en la misma línea. La UNALM perdió 20 años en los cuales pudo haberse consolidado su carrera de Ingeniería Biológica.

Como prueba de su perseverancia, en el año 2001 como Homenaje por el centenario de la UNALM, y con el apoyo y colaboración de sus colegas de la Facultad de Ciencias, de Industrias Alimentarias y profesores del extranjero, el doctor Gutiérrez impulsó la creación del Primer Programa Doctoral, en Ciencias e Ingeniería Biológicas (PDCIB), del cual fue Coordinador Académico hasta el año 2010. Desde el año 2016, el PDCIB cuenta con financiamiento del CONCYTEC (FONDECYTCIENCIA ACTIVA) para formar 4 promociones de doctores, con la más alta exigencia académica.

También fue organizador y Primer Coordinador de la oficina de Asesoría de Relaciones Internacionales, Proyectos y Propiedad Intelectual de la UNALM y el Primer Director de Oficina Rectoral de Gestión Internacional y Proyectos Institucionales.

La impecable trayectoria del Profesor Marcel GutiérrezCorrea en la UNALM incluyen 41 años de Investigación inin- 
terrumpida en Biotecnología Industrial. El mejor legado que deja el profesor Marcel es su calidad de Maestro ejemplar, su vocación sagrada por la enseñanza y su completa entrega a "su bien amada universidad".

Desde el año 2013 y hasta el momento de su partida trabajó incansablemente en el proyecto para la construcción del nuevo Laboratorio de Micología y Biotecnología (LMB) de la UNALM, con un área de aproximadamente $2000 \mathrm{~m} 2$ y con equipamiento de última generación en ingeniería genómica e ingeniería biológica, buscando colocarlo al nivel de los mejores laboratorios de Latinoamérica. Este nuevo edificio del LMB será inaugurado este año y en homenaje a su fundador, gestor y Director, será denominado en adelante Laboratorio de Micología y Biotecnología "Marcel Gutiérrez-Correa". Su nombre y su obra ya son parte de la historia de la UNALM y de la ciencia en el país.

La promoción 2017-I de la Universidad Nacional Agraria La Molina, lleva su insigne nombre, como Homenaje a su excelencia.

\section{Su trayectoria profesional y académica}

A lo largo de su trayectoria profesional, destacan muchos logros que pusieron en alto el nombre de su Alma Mater.

Como merecido reconocimiento, puede ser considerado como el "Padre de la Biotecnología en el Perü". En el año 1984 organizó el Primer Curso Nacional de Biotecnología, con asistencia masiva de investigadores de todo el Perú, producto del cual se publicó el libro "Principios de Biotecnología" con participación de varios profesores de la Facultad de Ciencias de la UNALM y de otras Universidades. En 1986, fue elegido Presidente de la Primera Comisión Nacional de Biotecnología, creada por el CONCYTEC, con la participación de 25 profesionales procedentes de UPCH, UNMSM, Sinquisa y UNALM. En el año 1987, dicha Comisión presentó el Primer Plan Nacional de Biotecnología a la Presidencia de CONCYTEC, con actividades de formación de recursos humanos, establecimiento de centros de excelencia, implementación de equipamiento científico y proyectos de investigación prioritarios, entre otras propuestas. Desafortunadamente, CONCYTEC no implementó dicho plan por razones políticas y recién después de más de una década, la Biotecnología fue considerada por el CONCYTEC, como línea de investigación prioritaria para el país.

En el año 1987, dicha Comisión presentó el Primer Plan Nacional de Biotecnología a la Presidencia de CONCYTEC, que incluía actividades de formación de recursos humanos, establecimiento de centros de excelencia, implementación de equipamiento científico y proyectos de investigación prioritarios, entre otras propuestas. Desafortunadamente, la Presidencia de la Institución no implementó dicho plan por razones políticas y recién después de más de una década, la Biotecnología fue reivindicada y considerada por el CONCYTEC como línea de investigación prioritaria para el país.

En su incansable labor de promoción de la biotecnología, fue asesor en Biotecnología Industrial para el Programa Bolivar / BID / Sociedad Nacional de Industrias, consultor de la Corporación Andina de Fomento (CAF), consultor del CONCYTEC, consultor del Ministerio de la Producción del Perú y del Banco
Interamericano de Desarrollo (BID).

También tuvo un aporte muy importante en la organización de programas de apoyo económico a la investigación en el país. Su asesoría al Programa INCAGRO en el año 2001 permitió establecer los conceptos, alcances e impactos esperados de la Investigación Básica y Estratégica, Investigación Aplicada e Investigación Adaptativa. Esta concepción fue recogida y aplicada para el otorgamiento de fondos concursables de INCAGRO, y posteriormente por FINCYT, CONCYTEC, INNOVATE, entre otros.

En el año 2007 fue nuevamente premiado por el Colegio de Biólogos del Perú y se le hizo una ceremonia de reconocimiento por sus "Servicios distinguidos al Perú en Ciencias Biológicas".

En ese mismo año, su biografía fue publicada en Who 's Who in Science and Engineering, 2007, 10th Edition.

En el 2008 y en mérito a su contribución científica al país, fue distinguido como Miembro Titular y Académico de Número de la Academia Nacional de Ciencias del Perú.

También fue premiado con la "Medalla de Oro del Cincuentenario" por la Universidad Nacional San Luis Gonzaga de Ica.

Su trayectoria internacional incluye el haber sido uno de los primeros científicos peruanos en ser elegido como Embajador para la Región Andina de la American Society for Microbiology (ASM, EEUU) para el período 2007 - 2010. Gracias a su excelente gestión de promoción del conocimiento en microbiología y biotecnología y habiendo logrado afiliar a más de mil nuevos estudiantes peruanos y latinoamericanos, fue premiado por la ASM como "Embajador del Año 2008".

En el año 2011 fue reconocido en una ceremonia realizada en Boston, por sus servicios distinguidos Outstanding Services to the American Society for Microbiology en una ceremonia realizada en Boston. En el mismo año 2011, fue elegido como Ambassador of the International Society of Microbial Ecology.

Finalmente, fue nuevamente condecorado por servicios distinguidos Outstanding Services to The American Society for Microbiology como ASM Ambassador to Perú 2010-2014.

Su desempeño como profesor universitario trascendió las fronteras de la UNALM, y fue invitado como profesor visitante en diferentes universidades del país, como la Universidad Nacional Mayor de San Marcos, Universidad Ricardo Palma, Universidad Peruana Cayetano Heredia, Universidad Nacional San Cristóbal de Huamanga, Universidad Nacional de Trujillo, Universidad Nacional San Antonio Abad del Cusco, Universidad de Piura, entre otras. En el extranjero fue profesor invitado en Colorado State University, Universidad Católica de Valparaíso, Universidad de la Península de Santa Elena (UPSE), entre otras.

$\mathrm{Su}$ aporte profesional también reflejó su compromiso con la difusión de la ciencia hacia la sociedad, representando brillantemente a la UNALM, en entrevistas de TV, radio, diarios, congresos, conferencias nacionales e internacionales, y en la elaboración de artículos de opinión y divulgación.

El doctor Marcel Gutiérrez-Correa fue uno de los primeros en el país en resaltar la importancia de la Bioecnonomía como nuevo modelo económico mundial, basado en el uso de recursos 
energéticos renovables y en la sustitución de la industria petroquímica por la industria biotecnológica. Advirtió tempranamente, que no podíamos ser ajenos a estos cambios y que el valor de nuestra biodiversidad radica principalmente en la riqueza de genes, que constituyen las fuentes primarias para la innovación tecnológica y desarrollo de nuevos procesos productivos de base biológica. Promovió el uso racional de la Biodiversidad a través de tecnologías de alto impacto. En este sentido, fue un convencido de los beneficios de la Biotecnología Moderna para el desarrollo económico y agrícola en el país, y los últimos años de su actividad profesional realizó una labor constante de docencia, difundiendo evidencia científica y objetiva sobre los beneficios y avances de esta tecnología por diferentes medios, incluyendo las redes sociales. Sus últimos artículos de divulgación fueron dedicados a la trilogía Bioeconomía, Biodiversidad y Biotecnología.

Por su trayectoria académica y producción científica en el año 2016 fue acreditado como investigador en la base de datos REGINA del CONCYTEC y calificado como Profesor Investigador por el MINEDU.

\section{Su legado como investigador}

Como se ha mencionado anteriormente, la vocación por la investigación científica del profesor Marcel, lo llevó a buscar muy tempranamente en su carrera docente los recursos necesarios que le permitieran iniciar su propia línea de investigación, para ese entonces, diferente a las desarrolladas en el departamento de Biología, ya que la Microbiología estaba fuertemente orientada a aspectos clínicos. Es así que en el año 1977, gracias al apoyo financiero del Centro de Investigaciones en Zonas Áridas y a su Director, el Dr. Carlos López Ocaña, forma junto a un grupo estudiantes el Laboratorio de Micología, que llevó esa denominación hasta el año 1987. A partir de ese año, ya habiendo

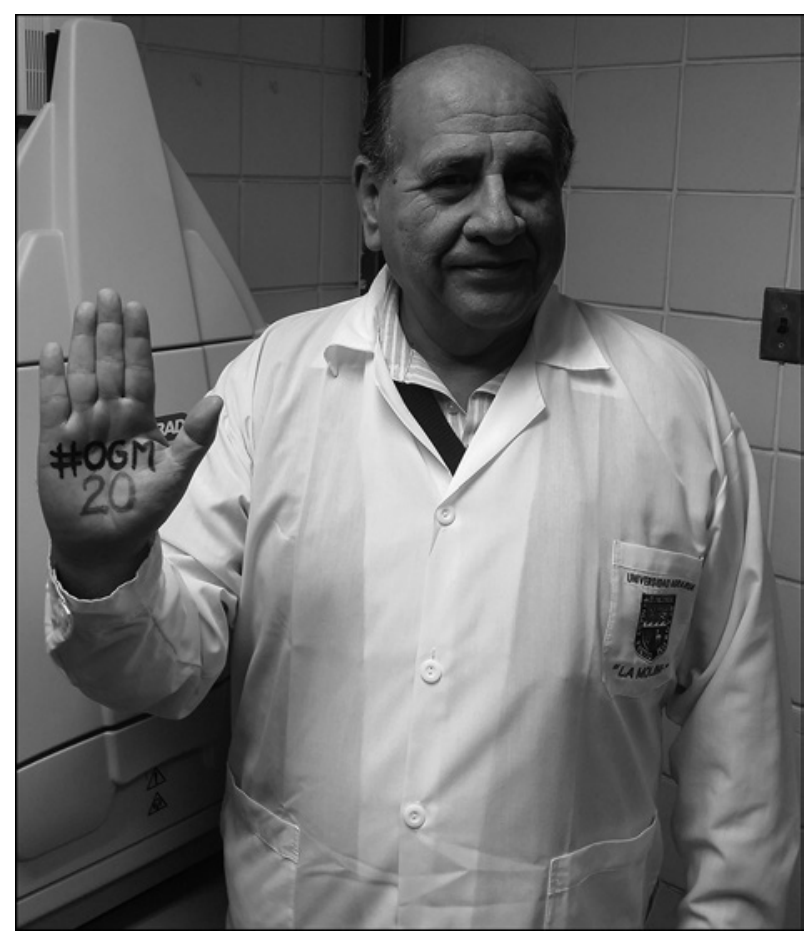

El Profesor Marcel Gutiérrez-Correa fue un convencido de los beneficios de la Biotecnología Moderna en el desarrollo económico y agrícola en el país. Su postura se resume en esta frase: "Los transgénicos no destruyen la biodiversidad, por le contrario ayudan a conservarla." ampliado sus instalaciones e implementado su infraestructura con equipos de última generación para biología molecular y biotecnología industrial, adopta el nombre de Laboratorio de Micología y Biotecnología (LMB), del cual fue Director hasta su partida, el 27 de Febrero del 2017.

Las líneas de investigación iniciales del Laboratorio de Micología durante el período 1982 - 1987 se centraron en la ecología microbiana, conjugando la microbiología clásica con el modelamiento matemático para generar los primeros estudios a nivel nacional sobre dinámica de poblaciones microbianas, que constan en los artículos científicos de esos años.

A partir de la siguiente década, se inició la investigación en Biotecnología Industrial, con énfasis en la producción de enzimas, con lo cual el LMB fue reconocido y considerado pionero y referente internacional por sus investigaciones y publicaciones en Fermentación en Estado Sólido y cultivos mixtos. La tesis doctoral del Profesor Marcel Toward the understanding offungal mixed culture solid substrate fermentation for cellulolytic enzyme production (1999) fue producto de ańos de investigación en el tema. En los últimos 15 años, desarrolló investigaciones pioneras en el uso de biopelículas de hongos filamentosos. Esto permitió proponer una nueva categoría de bioprocesos, La Fermentación por adhesión a Superficies (Gutierrez-Correa $\&$ Villena 2003) e introducir un nuevo enfoque científico en la comunidad académica mundial que ha permitido entender que las biopelículas de hongos son una condición real, natural y compleja de crecimiento de estos organismos, y que constituyen sistemas altamente relevantes en la medicina y la industria. A partir del año 2006 a raíz de las primeras publicaciones mundiales del LMB en el tema, diferentes grupos de investigación en Escocia, Brasil, Canadá, India y otros países, adoptaron esta nueva propuesta y vienen desarrollando investigaciones sobre biopelículas de hongos de relevancia clínica e industrial. Nuevamente, el LMB, se convirtió en referente mundial. Los resultados en este campo permitieron iniciar en el país en el año 2008, la investigación en Genómica funcional, habiendo publicado el primer artículo en el mundo sobre proteómica de biopelículas de Aspergillus niger.

Paralelamente, hacia el año 2008 el LMB inició en el país la investigación en Bioprospección Molecular y Metagenómica. Asimismo, en los últimos 5 años se ejecutaron bajo su dirección proyectos de Biología Sintética e Ingeniería Genómica para la obtención de factorías celulares de levaduras, y de edición génica de hongos mediante el sistema CRISPR/cas, entre otros.

En los últimos 2 años, el profesor Marcel había promovido el secuenciamiento de 10 genomas de hongos nativos, y tiene dos publicaciones póstumas sobre ello.

En su labor de Director y apoyado por su grupo de investigación, estableció las líneas de investigación del LMB hasta el año 2021: a) Ingeniería Genómica, con énfasis en metagenómica y bioprospección molecular, genómica funcional y biología sintética; b) Ingeniería Biológica para el desarrollo de bioprocesos derivados de la ingeniería genómica. En los últimos 24 años ganó 17 proyectos de investigación y contratos de investigación de entidades y empresas nacionales e internacionales por aproximadamente 4 millones y medio de dólares. También transfirió una tecnología de producción de enzimas a una empresa peruana.

Gracias a su gestión, el LMB es reconocido por organizacio- 
nes internacionales como la American Society for Microbiology, Society for Applied Microbiology, US Department of Agriculture, International Society for Food, Agriculture and Environment y la Academy Science Society.

\section{Su producción científica}

Su contribución científica abarca más de 90 publicaciones, incluyendo artículos científicos, revisiones (reviews) y capítulos de libros, entre otros.

\section{Artículos científicos}

Sujay Paul, Angel Zhang, Yvette Ludeña, Gretty K. Villena, Fengan Yu, David H. Sherman, Marcel Gutiérrez-Correa. 2017. Insights from the genome of a high alkaline cellulase producing Aspergillus fumigatus strain obtained from Peruvian Amazon rainforest. Journal of Biotechnology 251:53-58. Publicación póstuma

Sujay Paul, Angel Zhang, Yvette Ludeña, Gretty K. Villena, Fengan Yu, David H. Sherman, Marcel Gutiérrez-Correa. 2017. Insights from the genome of a high alkaline cellulase producing Aspergillus fumigatus strain obtained from Peruvian Amazon rainforest. Journal of Biotechnology 251:53-58. Publicación póstuma.

Paul, Sujay, Yolanda Cortez, Nadia Vera, Gretty K. Villena, and Marcel Gutiérrez-Correa. 2016. Metagenomic analysis of microbial community of an Amazonian geothermal spring in Peru. Genomics Data 9: 63-66.

Paul, Sujay, Yolanda Cortez, Nadia Vera, Gretty K. Villena, and Marcel Gutiérrez-Correa. 2016. Metagenomic Analysis of Microbial Communities in the Soil-mousse Surrounding of an Amazonian Geothermal Spring in Peru. British Biotechnology Journal 15(1): 1-11, 2016, Article no.BBJ.27519, DOI: $10.9734 / \mathrm{BBJ} / 2016 / 27519$

Cortez, Yolanda, Sujay Paul, Gretty K. Villena and Marcel GutiérrezCorrea. 2016. Isolation and Characterization of Cellulase Producing Bacterial Strains from an Amazonian Geothermal Spring in Peru. British Microbiology Research Journal

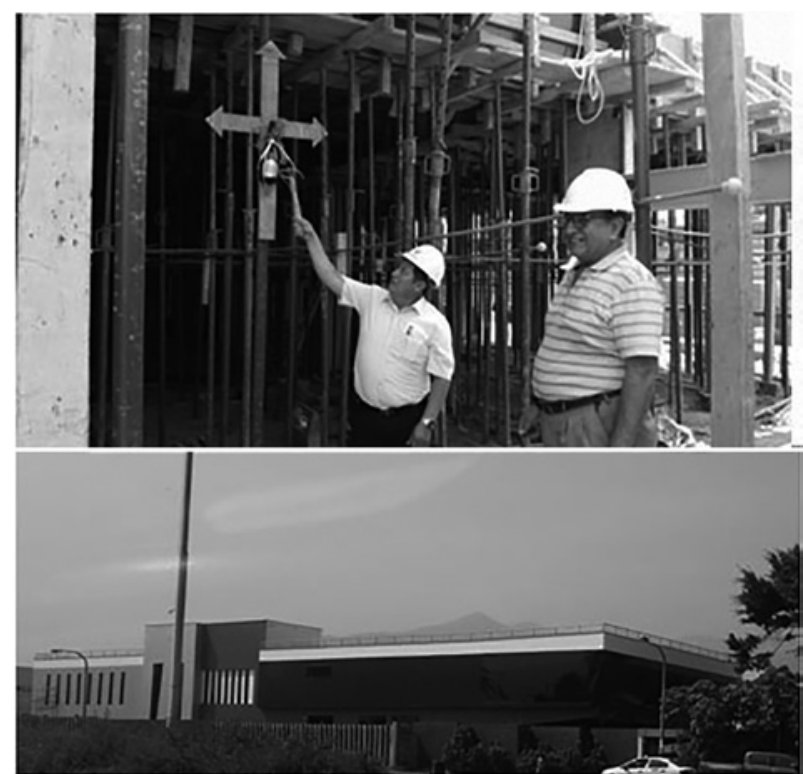

El doctor Marcel Gutiérrez - Correa fue gestor en el año 2013 de la construcción del nuevo Laboratorio de Micología y Biotecnología de la UNALM. En sus últimos años de labor, realizó el seguimiento y supervisión de la obra que lamentablemente no llegó a inaugurar. En la fotografía, aparece junto al entonces Rector de la UNALM, Dr. Abel Mejía, durante el techado del primer piso del edificio. El nuevo LMB (abajo) será inaugurado en el presente año, y a iniciativa del grupo de investigación que lo acompañó y del actual Rector de la UNALM, Dr. Enrique Flores, llevará el nombre de Laboratorio de Micología y Biotecnología "Marcel Gutiérrez - Correa" en Homenaje a quién fuera su Director.
15(5): 1-8, 2016, Article no.BMRJ.27520, DOI: $10.9734 /$ BMRJ/2016/27520

Cerrón, Luis M., David Romero-Suárez, Nadia Vera, Yvette Ludeña, Gretty K. Villena and Marcel Gutiérrez-Correa. 2015. Decolorization of textile reactive dyes and effluents by biofilms of Trametes polyzona LMB-TM5 and Ceriporia sp. LMB-TM1 isolated from the Peruvian rainforest. Water, Air and Soil Pollution 226, 235 (DOI 10.1007/ s11270-015-2505-4).

Vega, Karin, Victor H. Sarmiento, Yvette Ludeńa, Nadia Vera, Carmen Tamariz-Angeles, Gretty K. Villena and Marcel Gutiérrez-Correa. 2015. Alkaline cellulase production by Penicillium mallochii LMB-HP37 isolated from soils of a Peruvian rainforest. British Biotechnology Journal 7(4), 160-168.

Tamariz-Angeles, Carmen, Percy Olivera-Gonzales, Gretty K. Villena and Marcel Gutiérrez-Correa. 2014. Isolation and identification of cellulolytic and xylanolytic bacteria from Huancarhuaz hot spring, Peru. Annual Research and Review in Biology 4(19), 2920-2930.

Gutiérrez-Correa, Marcel and Gretty K. Villena. 2012. Batch and repeated batch cellulase production by mixed cultures of Trichoderma reesei and Aspergillus niger or Aspergillus phoenicis. Journal of Microbiology and Biotechnology Research 2(6), 929-935.

Vega, Karin, Gretty K. Villena, Victor H. Sarmiento, Yvette Ludeña, Nadia Vera and Marcel Gutiérrez-Correa. 2012. Production of alkaline cellulase by fungi isolated from an undisturbed rain forest of Peru. Biotechnology Research International Volume 2012, Article ID 934325, 7 pages, doi: $10.1155 / 2012 / 934325$.

Gretty K. Villena and Marcel Gutiérrez-Correa. 2012. Kinetic analysis of Aspergillus niger cellulase and xylanase production in biofilm and submerged fermentation. Journal of Microbiology and Biotechnology Research 2, 805-814.

Gutiérrez-Correa, Marcel, Yvette Ludeña, Gordon Ramage and Gretty K. Villena. 2012. Recent advances on filamentous fungal biofilms for industrial uses. Applied Biochemistry and Biotechnology 167,1235-1253.

Rueda, Analiz, Cecilia Sifuentes, Robert H. Gilman, Andrés H. Gutiérrez, Ruby Piña, Nancy Chile, Sebastián Carrasco, Sandra Larson, Holger Mayta, Manuela Verástegui, Silvia Rodriguez, Marcel Gutiérrez-Correa, Héctor H. García, Patricia Sheen, Mirko Zimic. 2011. TsAg5, a Taenia solium cysticercus protein with a marginal trypsin-like activity in the diagnosis of human neurocysticercosis. Molecular and Biochemical Parasitology 180, 115-119.

Ramage, Gordon, Ranjith Rajendran, Marcel Gutiérrez-Correa, Brian Jones and Craig Williams. 2011. Aspergillus biofilms: clinical and industrial significance. FEMS Microbiology Letters 324, 89-97.

Villena, G.K. \& M. Gutiérrez-Correa. 2011. Assessment of Aspergillus niger biofilm growth kinetics in minibioreactors by carbon dioxide evolution. African Journal of Biotechnology 10(62): 13495-13504.

Jiménez, Martha V., Gabriella G. Zarkovic, Gretty K. Villena \& Marcel Gutiérrez-Correa. 2011. The effect of bead diameter and cell density in the ethanol production by alginate immobilized yeast. [Efecto del diámetro de esfera y la densidad celular en la producción de etanol con levadura inmovilizada en alginato]. Revista Colombiana de Biotecnología 13(1), 8-15.

Izarra, Myriam L., Mónica L. Santayana, Gretty K. Villena \& $\underline{\text { Marcel }}$ Gutiérrez-Correa. 2010. Influence of inoculum concentration on Aspergillus niger morphology [Influencia de la concentración de inóculo en la morfología para la producción de celulasa y xilanasa por Aspergillus niger]. Revista Colombiana de Biotecnología 12(2), 139-150.

Gamarra, Norma N., Gretty K. Villena y Marcel Gutiérrez-Correa. 2010. Cellulase production by Aspergillus niger in biofilm, solid-state and submerged fermentations. Applied Microbiology and Biotechnology 87, 545-551.

Villena, Gretty K., Takashi Fujikawa, Shinji Tsuyumu y Marcel Gutiérrez-Correa. 2010. Structural analysis of biofilms and pellets of Aspergillus niger by confocal scanning laser microscopy and cryo scanning electron microscopy. Bioresource 
Technology 101, 1920-1926.

Villena, G.K., L. Venkatesh, A. Yamazaki, S. Tsuyumuy M. GutiérrezCorrea. 2009. Initial intracellular proteome profile of Aspergillus niger biofilms. Revista Peruana de Biología 16, 101-108.

Villena, G.K., T. Fujikawa, S. Tsuyumu, \& M. Gutiérrez-Correa. 2009. Differential gene expression of some lignocellulolytic enzymes in Aspergillus niger biofilms. Revista Peruana de Biologia 15, 97-102.

Villena, G.K. \& M. Gutiérrez-Correa. 2007. Morphological patterns of Aspergillus niger biofilms and pellets related to lignocellulolytic enzyme productivities. Letters in Applied Microbiology, 45, 231-237.

Villena, G.K. \& M. Gutiérrez-Correa. 2007. Production of lignocellulolytic enzymes by Aspergillus niger biofilms at variable water activities. Electronic Journal of Biotechnology, Vol.10 No.1, Issue of January 15, 2007, DOI: 10.2225/vol10issue5-fulltext-2.

Villena, G.K. \& M. Gutiérrez-Correa. 2006. Production of cellulase by Aspergillus niger biofilms developed on polyester cloth. Letters in Applied Microbiology 43, 262 -268.

Gutiérrez-Correa, M. 2003. Opinion on surface adhesión fermentation. Agri-Food "Research and News" 1(1), 19.

Gutiérrez-Correa, Marcel \& Gretty K. Villena. 2003. Surface Adhesion Fermentation: A new fermentation category. Revista Peruana de Biología 10(2), 113-124.

Villena, Gretty, \& Marcel Gutiérrez-Correa. 2003. Aspergillus niger biofilms for cellulase production: some structural and physiological aspects [Biopelículas de Aspergillus niger para la producción de celulasas: algunos aspectos estructurales y fisiológicos]. Revista Peruana de Biología 10(1), 78-87.

Villena G., P. Moreno \& M. Gutiérrez-Correa. 2001. Cellulase production by fungal biofilms on polyester cloth. Agro-food-Industry Hi-Tech 12, 32-35, January/February.

Gutiérrez-Correa, M. 1999. Toward the understanding of fungal mixed culture solid substrate fermentation for cellulolytic enzyme production. Ph.D. Thesis. Gifu University

Gutiérrez-Correa, M. \& R.P. Tengerdy. 1999. Cellulolytic enzyme production by fungal mixed solid substrate fermentation. Agro-food-Industry Hi-Tech 10, 6-8, March/April

Gutiérrez-Correa, M., L. Portal, P. Moreno \& R.P. Tengerdy. 1999. Mixed culture solid substrate fermentation of Trichoderma reesei with Aspergillus niger on sugar cane bagasse. Bioresource Technology 68 (2), 173-178. ]

Gutiérrez-Correa, M. \& R.P. Tengerdy. 1998. Xylanase production by fungal mixed culture solid substrate fermentation on sugar cane bagasse. Biotechnology Letters 20, 45-47.

Gutiérrez-Correa, M. \& R.P. Tengerdy. 1997. Production of cellulase on sugar cane bagasse by fungal mixed culture solid substrate fermentation. Biotechnology Letters 19, 665-667.

Meza, V., P. Moreno, R.P. Tengerdy \& M. Gutiérrez-Correa. 1995. Transfer of a benomyl resistance marker by heat-inactivated Trichoderma reesei protoplasts. Biotechnology Letters 17, 827-832.

Dueñas, R., R.P. Tengerdy \& M. Gutiérrez-Correa. 1995. Cellulase production by mixed fungi in solid substrate fermentation of bagasse. World Journal of Microbiology and Biotechnology 11, 333-337.

Castillo, M.R., M. Gutiérrez-Correa, J.C. Linden \& R.P. Tengerdy. 1994. Mixed culture solid substrate fermentation for cellulolytic enzyme production. Biotechnology Letters 16, 967-972.

Flores, J.J., M. Gutiérrez-Correa \& R.P. Tengerdy. 1994. Citric acid production by solid substrate fermentation of prickly pear peel with Aspergillus niger. Agro-Food-Industry Hi-Tech, 5, 18-20 (Jan./Feb.).

Muñiz, G., M. Gutiérrez-Correa \& R.P. Tengerdy. 1993. Solid substrate fermentation of potato cellulosic residues with Chaetomium cellulolyticum. Agro-Food-Industry Hi-Tech, 4, 33-34 (Jan./Feb.).

Ceroni, A. y M. Gutiérrez-Correa. 1988. Producción de celulasas por hongos: estudios cinéticos en hongos silvestres. Boletín de Lima (55), 13-20. [2 citaciones]

Ceroni, A. y M. Gutiérrez-Correa. 1987. Determinación de la capacidad celulolítica de hongos de suelo. Biota 12(94),2-12.

Julca, M. y M. Gutiérrez-Correa. 1987. Producción de celulasas por hongos: Obtención de mutantes hipercelulolíticos. Boletin de Lima (54),48-54

Márquez, A.J. y M. Gutiérrez-Correa. 1984. Biodeterioro de textiles arqueológicos. Boletín de Lima (34),77-83.

Gutiérrez-Correa, M. y J. Jhoncon. 1982/1985. Estudios microecológicos de los suelos de las lomas de Lachay (Perú). I. Dinámica poblacional de microhongos. Anales Científicos UNA 21,33-39.

Nakarino, C., J. Jhoncon y M. Gutiérrez-Correa. 1982/1985. Inhibición de enterobacterias en suelo regado con aguas especialmente tratadas. Anales Cientificos UNA 21,25-32.

Gutiérrez-Correa, M. y J. Jhoncon. 1983. Estudios microecológicos de los suelos de las lomas de Lachay (Perú). IV. Hongos celulolíticos. Zonas Aridas (3),103-105.

Moreno, Patricia y M. Gutiérrez-Correa. 1983. Estudios microecológicos de los suelos de las lomas de Lachay (Perú). III. Dinámica poblacional de hongos rizosféricos. Zonas Aridas (3),99-102.

Jhoncon, J. y M. Gutiérrez-Correa. 1983. Ecología de Penicillium en los suelos de las lomas de Lachay. III. Descripción de algunas especies. Zonas Aridas (3),95-98.

Jhoncon, J. y M. Gutiérrez-Correa. 1983. Ecología de Penicillium en los suelos de las lomas de Lachay. II. Distribución. Zonas Aridas (3),91-94.

Gutiérrez-Correa, M. y J. Jhoncon. 1982. Ecología de Penicillium en los suelos de las lomas de Lachay. I. Dinámica poblacional. Zonas Aridas (2),87-91.

Zuñiga, D. y M. Gutiérrez-Correa. 1982. Dinámica poblacional de diazotrofos libres fijadores de nitrógeno en la rizósfera de Sicyos baderoa. Zonas Aridas (2),79-86.

Ihoncon, J. y M. Gutiérrez-Correa. 1982. Estudios microecológicos de los suelos de las lomas de Lachay (Perú). II. Microhongos. Zonas Aridas (1),81-86.

Yague, N. y M. Gutiérrez-Correa. 1982. Dinámica poblacional de las bacterias del ciclo del nitrógeno de las lomas de Lachay. Zonas Aridas (1),75-80.

Gutiérrez-Correa, M. y D. Zuñiga. 1977. Aislamiento de Saprolegnia y hongos Saprolegniales por la "técnica de microvacuum". Anales Cientificos UNA 15,191-194.

Díaz, M., C.J. Galecio y M. Gutiérrez-Correa. 1977. Estudios sobre la contaminación bacteriana en productos lácteos de Piura. II. Queso de pasta blanda (mantecoso). Anales Científicos UNA 15,39-41.

Diaz, M., P. Nuñez y M. Gutiérrez-Correa. 1977. Estudios sobre la contaminación bacteriana en productos lácteos de Piura. I. Leche envasada en bolsas de plástico. Anales Cientificos UNA 15,35-38.

Gutiérrez-Correa, M. y L. Basto. 1977. El agua de coco como sustrato para la producción de levadura-alimento. Anales Científicos UNA 15,29-34.

Gutiérrez-Correa, Marcel. 1975. Estudio del antagonismo entre Fusarium sp y Helminthosporium sativum in vitro: Influencia del sustrato y la temperatura. Anales Cientificos UNA 13,23-29.

\section{Capítulos de Libros y monografías}

Gutiérrez-Correa, Marcel \& Gretty K. Villena. 2010. Characteristics and techniques of fermentation systems. In "Comprehensive Food Fermentation and Biotechnology". Ashok Pandey, Carlos Ricardo Soccol, Christian Larroche, Edgard Gnansounou, Claude-Gilles Dussap (eds.). Volume I, Chapter 7, p.183227. Asiatech Publisher, Inc. New Delhi.

Gutiérrez-Correa, Marcel. 2010. Megaproyecto de Biotecnología Biodiversidad, Biotecnología y Bioeconomía: Valorización biotecnológica de la biodiversidad. En "Identificación de Megaproyectos de Investigación Cientifica”. J. Segovia-Juárez, ed., p.21-39. CONCYTEC (ISBN: 978-9972-50-137-1), Lima.

Villena G.K., Gutiérrez-Correa M., Ludeña Y. \& G. Sandoval. 2010. Genómica Funcional Microbiana: Guía de Laboratorio. LMB-UNALM/FINCyT, Lima, Perú, Agosto

Gutiérrez-Correa M., Villena G.K., Vera N., Ludeña Y. \& G. Sandoval. 2010. Bioprospección Molecular: Guía de Laboratorio. LMB-UNALM/FINCyT, Lima, Perú, Setiembre.

Gutiérrez-Correa, M. \& R. Estrada. 2008. Línea base para la implementación del programa nacional en biotecnología agraria y agroindustrial en el Perú. En "Lineas de Base para la Implementación de Programas Estratégicos” (H. Fano \& M. 
Torres, eds.), pp. 121-140, INCAGRO (ISBN: 978-60345061-4-5), Lima.

Gutiérrez-Correa, M. 2005. Ciencias Biológicas, Bioquímica, Biología Molecular y Biotecnología en el Perú. En "La Investigación Cientifica y Tecnológica en el Perú", CONCYTEC, Volumen I (ISBN 9972-53-046-9), pp.264-343, BCR-CONCYTEC, Lima.

Gutiérrez-Correa, Marcel .1996. Fermentación en Sustrato Sólido en Cultivo Mixto. En V Curso Latinoamericano de Biotecnología. 25pp., Escuela de Ingeniería Bioquímica/Universidad Católica de Valparaíso: Valparaíso, Chile.

Gutiérrez-Correa, Marcel .1996. Genética Microbiana. En V Curso Latinoamericano de Biotecnología. pp.27-39, Escuela de Ingeniería Bioquímica/Universidad Católica de Valparaíso: Valparaíso, Chile.

Gutiérrez-Correa, Marcel .1996. Fisiología y Control Metabólico en Microorganismos. En V Curso Latinoamericano de Biotecnología. pp.9-25, Escuela de Ingeniería Bioquímica/Universidad Católica de Valparaíso: Valparaíso, Chile.

Gutiérrez-Correa, Marcel. 1994. La Biotecnología en el Perú. En Informe de las Mesas Redondas sobre Biotecnologia, Energias Renovables, Nuevos Materiales y Tecnología de la Información. pp.62-82, Proyecto de Monitoreo de Nuevas Tecnologías (ALA/89/13), Universidad Andina Simón Bolivar/Comisión Europea:Santa Cruz, Bolivia.

Gutiérrez-Correa, Marcel (Director). 1992. Biotecnología Aplicada a la Agricultura. 39pp, LMB/UNALM:Lima.

IICA. 1991. Guides for the Release to Environment of Geneticaly Modified Organisms. 145pp., IICA: San José [como colaborador]

IICA. 1988. Guides for the Use and Safety of the Techniques of Genetic Engineering or Recombinant DNA Technology. 151pp., IICA/ DRE/EUA/88/001: Washington, D.C. [como colaborador]

Gutiérrez-Correa, Marcel. 1987. Bioconversión de residuos lignocelulósicos en etanol y proteína. En Ciencia y Tecnología para el Desarrollo Nacional, pp.81-95, CONCYTEC: Lima.

Gutiérrez-Correa, Marcel. 1986. Producción de Etanoly Proteina. 45pp., CONCYTEC:Lima. Monogr. [1 citación]

Gutiérrez-Correa, Marcel. 1986. Biotecnología: Conceptos y Lineas Futuras. 38pp., CONCYTEC:Lima.

Gutiérrez-Correa, Marcel y Robert P. Tengerdy. 1985. Tópicos Especiales en Fisiología Microbiana y Biotecnología: Guía de Laboratorio. 20pp., SPM:Cusco.

Gutiérrez-Correa, Marcel (Director). 1985. Principios de Biotecnología. 293pp., UNALM:Lima.

Gutiérrez-Correa, Marcel. 1984. Aprovechamiento de Residuos Celulósicos, CONCYTEC:Lima.

Gutiérrez-Correa, Marcel. 1983. Biología General: Guia de Estudio. 246pp., UNALM:Lima.

\section{Artículos de Divulgación}

Gutiérrez-Correa, Marcel. 2012. El camino de la biotecnología en la Universidad Nacional Agraria La Molina. Revista Peruana de Biología 19(3), 355-364.

Maloy, Stanley \& Marcel Gutiérrez-Correa. 2011. ASM Participation in Peruvian Minicourses. Microbe 6(5), 244-245. May

Gutiérrez-Correa, Marcel. 2009. El Valor Económico de los Genes: Una Visión Preliminar. Bios 2(3), 4-7.

Gutiérrez-Correa, Marcel. 2009. ASM in Ecuador: Strengthening Ties with Latin America. Microbe 4(1), 36.

Gutiérrez-Correa, Marcel. 2009. Bioeconomía (Segunda Parte). Revista Agronegocios 3(1), 16 - 19.

Gutiérrez-Correa, Marcel. 2008. ASM in Peru: Supporting Latin America’s Youth. Microbe 3(6), 298-299.

Gutiérrez-Correa, Marcel. 2008. Bioeconomía (Primera Parte). Revista Agronegocios 2(3), 14 - 17.

Gutiérrez-Correa, Marcel. 2008. Presente y futuro de los alimentos humanos de origen transgénico. Anales de la Academia $\mathrm{Na}$ cional de Medicina, p. 63 - 73.

Villena, G.K. \& M. Gutiérrez-Correa. 2008. Genómica funcional de hongos filamentosos. Bios 1(1), 28 - 31.

Gutiérrez-Correa, Marcel. 2008. Bioeconomía: la economía del Siglo XXI. Bios 1(1), 3 - 6 .
Gutiérrez-Correa, Marcel. 2007. Biotecnología, Biodiversidad y Bioeconomía. Perú Económico, Enero, pp. 26-27.

Gutiérrez-Correa, Marcel. 2006. Book review: Essential Fungal Genetics (David Moore and LilyAnn Novak Frazer. Springer-Verlag, New York. 2002, 357p + xi, 59 figs. ISBN 0-387-95367-1). Microbiologist 7(2), 53. June.

Gutiérrez-Correa, Marcel. 2002. Bioprospecting, Genomics and Biotechnology, En Libro de Resúmenes I Congreso Internacional de Ciencias Farmacéuticas y Biotecnologia, pp.20-22. UCSM, Arequipa

Gutiérrez-Correa, Marcel y Patricia Moreno. 1992. Agriculture in Peru. Agro-Food-Industry Hi-Tech, 3:35-37(September/October).

Gutiérrez-Correa, Marcel. 1992. Biotecnología y agricultura II. AgroEnfoque (50), 22,24.

Gutiérrez-Correa, Marcel. 1992. Biotecnología y agricultura. AgroEnfoque (48), 12-14.

Gutiérrez-Correa, Marcel. 1988. Biotecnología Convencional y de Punta y sus aplicaciones. Anales de la Academia Peruana de Farmacia 3, 30-49.

Gutiérrez-Correa, Marcel. 1986. Bioconversión de residuos lignocelulósicos. Resúmenes del VIII Congreso Nacional de Biología, pp.4-5, CBP: Lima.

Gutiérrez-Correa, Marcel. 1985. Barbara McClintock y la tecnología del ADN Recombinante. Boletin de Lima (40),87-93.

\section{Artículos de Opinión}

Gutiérrez-Correa, Marcel. 2014. Otra víctima de la moratoria a los transgénicos: el agricultor algodonero. Negocios Internacionales 17(203): 22-25.

Gutiérrez-Correa, Marcel. 2013. Lo que perdemos con la moratoria a los transgénicos: El caso del maíz amarillo duro. Negocios Internacionales 16(188): 28-30.

Gutiérrez-Correa, Marcel. 2012. ¿Hay sustento científico para la moratoria de los transgénicos? No hay sustento científico. Agrum 11(39): 22.

Gutiérrez-Correa, Marcel. 2012. ¿La moratoria a los transgénicos reglamentada? Negocios Internacionales 15(183/184): 2223. Diciembre.

Gutiérrez-Correa, Marcel. 2011. Transgénicos: ¿Apocalípsis o revolución? Semanario COMEXPERU No 642: 5. Diciembre.

Gutiérrez-Correa, Marcel. 2011. De transgénicos y culantro o la guerra de los mundos. Semanario COMEXPERU No 634: 4. Octubre.

Gutiérrez-Correa, Marcel. 2011. Los transgénicos y los estados de ánimo. Semanario COMEXPERU No 627: 6. Setiembre.

Gutiérrez-Correa, Marcel. 2011. Transgénicos y el desarrollo sostenible. Negocios Internacionales 14(169): 12-13. Setiembre.

Gutiérrez-Correa, Marcel. 2011. Los transgénicos sí son necesarios. Negocios Internacionales 14(168): 20-21. Agosto.

Gutiérrez-Correa, Marcel. 2011. Los transgénicos y Marca Perú no se contraponen. Negocios Internacionales 14(166): 32-33. Junio.

Gutiérrez-Correa, Marcel. 2011. La desinformación sobre los transgénicos. Negocios Internacionales 14(165): 24-25. Mayo.

\section{Su actividad editorial y Membresías}

Como académico reconocido fue miembro de comité editorial de la Revista Peruana de Biología, UNMSM, Perú. También fue editor asociado, del Global Research Journal of Microbiology, Global Research Journals, 2012. Editor del Journal of the Selva Andina Research Society, Bolivia. Editor de The Scientific World Journal, Biotechnology Domain, Hindawi Publishing Corporation. Editor regional del Journal of Food, Agriculture and Environment.

Asimismo, fue evaluador de numerosos artículos científicos en varias revistas: African Journal of Biotechnology, African Journal of Microbiology Research, Agro-food Industry Hi-Tech, Applied Biochemistry and Biotechnology, Applied and Environmental Microbiology, Applied Microbiology and Biotechnology, Applied 


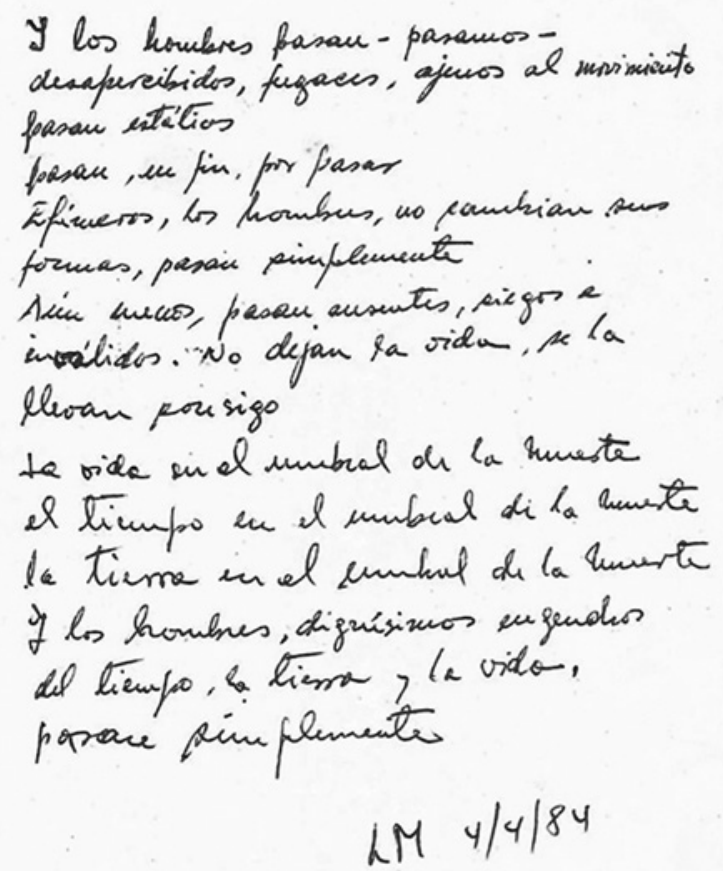

Ylos hombres pasan-pasamos

desapercibidos, fugaces, ajenos almovimiento

pasan estáticos

Pasan, en fin, por pasar

Efimeros, los hombres nocambian sus

formas, pasan simplemente

Aún menos, pasan ausentes, ciegose

inválidos. No dejan la vida, se la

llevan consigo

La vida en el umbral de lamuerte

el tiempo en el umbral delamuerte

la tierra en el umbral de la muerte

Ylos hombres, dignísimos engendros

del tiempo, la tierra y lavida,

pasan simplemente.

Marcel Gutiérrez-Correa. La Molina 4/4/84

Poema inédito del Doctor Marcel Gutiérrez-Correa, que junto a otros poemas escritos en idioma inglés y español, muestran el talento literario de nuestro reconocido Maestro.

Surface Science, Biochemical Engineering Journal, Biomass and Bioenergy, BioMed Research International, Bioprocess and Biosystems Engineering, Bioresource Technology, BMC Microbiology, Canadian Journal of Chemical Engineering, Chemical Engineering Journal, Electronic Journal of Biotechnology, Energy \& Fuels, Engineering in Life Sciences, Fungal Genetics and Biology, Journal of Agricultural Science and Technology, Journal of Applied Microbiology, Journal of Chemical Technology and Biotechnology, Journal of Microbiology and Biotechnology, Journal of Mycology, Journal of the Taiwan Institute of Chemical Engineers, Microbial Cell Factories, Microbiological Research, Mycopathologia, Process Biochemistry, Revista Peruana de Biologia, The Canadian Journal of Chemical Engineering, The Scientific World JOURNAL, Waste Management \& Research, Water Science and Technology.

En el círculo académico internacional fue Miembro de las siguientes Instituciones y Sociedades científicas:

- American Society for Microbiology (desde 2004), USA, Division O: Fermentation \& Biotechnology.

- Society for Applied Microbiology (desde 2004), UK.

- International Society of Food, Agriculture and Environment, Finland. Chair: Food Biotechnology Sub-section (desde 2002).

- $\quad$ European Federation of Biotechnology (desde 2004).
- Federation of European Microbiological Societies (desde 2006).

- International Organisation for Biotechnology and Bioengineering (desde 2005).

- Academy Science Society (desde 2010), Board and Award Committee member.

\section{El Poeta}

El arte no pasó desapercibido para el Dr. Marcel GutiérrezCorrea, mostrando particular talento para la poesía. En la década del 80 y durante su estadía en Rutgers University (EEUU), donde realizó sus estudios de Maestría, publicó numerosos poemas tanto en idioma inglés como español. Su referente literario fue su hermano Miguel Gutiérrez, reconocido escritor nacional. En uno de sus poemas inéditos, refleja su filosofía de vida y la modestia con la que asumió su paso por este mundo. Pero su vida no pasó desapercibida, dejó una huella imborrable e imperecedera. Su nombre y su obra jamás se olvidarán.

\section{Referencias consultadas}

Gutiérrez-Correa, Marcel. 2012. El camino de la biotecnología en la Universidad Nacional Agraria La Molina. Revista Peruana de Biología 19(3), 355-364.

Homenaje Académico a nuestro distinguido profesor Marcel Gutierrez Correa. Revsita AGRUM. Edición 52, Abril de 2017. 4-6.

Página Academia. edu. del Profesor Marcel Gutiérrez Correa (https:// lamolina.academia.edu/MarcelGuti\%C3\%A9rrezCorrea) 